\title{
Clinical Comparative Study Between Dexmedetomidine, Magnesium sulphate and Ketofol versus Midazolam for Sedation During Awake Fiberoptic Nasotracheal Intubation in Predicted Difficult Air ways Patients
}

\author{
Mostafa I. Shalaby, Maamoun M. Ismael, Ahmed S.A. Nasr, Mohammad H.M. Alqassas \\ Anathesia and Intensive Care Department, Faculty of Medicine, Al-Azhar University, Cairo, Egypt \\ Corresponding author: Mohammad H.M. Alqassas, Mobile: 01129939569; Email: dr.qassas1984@gmail.com
}

\begin{abstract}
Background and objectives: Awake fiber optic intubation (AFOI) is recommended technique in securing the airway in predicted difficult airway patients with sedation. However, it is not easy to achieve a comfortable sedation so conscious sedation is the key for a successful AFOI. The goal of the study to compare different conscious sedation strategies aimed to improve comfort and safety in patients prepared for elective surgeries. Subjects and Methods: Case control, randomized controlled study in predicted difficult airway patients scheduled for elective surgery under GA carried out at AL-Azhar University Hospitals after approval by the local ethical committee. 120 patients of age group 18-60 years old with ASA I and II prepared for nasal (AFOI) under conscious sedation after giving their informed written consent to participate in our study. The patients were randomly assigned into 4 groups: Group dexmedetomidine (DEX), group ketofol, group magnesium sulfate and group midazolam. HR, MAP, Oxygen saturation and end tidal $\mathrm{CO} 2$ were monitored. Sedation score, patient tolerance, patient satisfaction and intubation score (vocal cord movement and coughing) were assessed.

Results: All patients were successfully intubated by fiber optic and none of them developed bradycardia or reduced MAP more than 20\% from the base line during intubation. Group DEX mild decrease in MAP and HR ( $<10 \%$ fall when compared with the baseline value) after loading of drug and during intubation in contrast to midazolam, ketofol and magnesium sulphate, which increase during intubation. There was no statistically significant difference in the intubation scores, grimace score, time of intubation, number of attempts and saturation in between the groups with one episode of desaturation in group ketofol and two in group midazolam $(\mathrm{P}>0.05)$. Group ketofol and midazolam patients were sedated deeper after the start of the study drugs than group DEX and none of the patients were sedated to a score of $<2$ (modified OAA/S score) in either of the groups. Group magnesium showed lighter sedation level significantly different with other groups.

Conclusion: Study showed DEX provides optimum sedation without compromising airway or hemodynamic stability with favorable intubation time and less intubation attempts during AFOI in comparison to magnesium sulphate, ketofol and midazolam patients with better patient tolerance and satisfaction.
\end{abstract}

Keywords: (AFOI) Awake fiberoptic intubation, (DI) difficult intubation, (DEX) dexmedetomidine.

\section{INTRODUCTION}

Fiber optic intubation is a valuable technique in securing the airway in predicted difficult intubation scenario, compromised airway, lower airway pathology and when neck extension is to be avoided ${ }^{(I)}$.

Awake fiber optic intubation (AFOI) is used in patients with predicted difficult airway management. This study aimed to compare different conscious sedation strategies in order to improve comfort and safety in patient prepared for elective surgeries. In awake fiber optic intubation under intravenous (IV) sedation patient should remain calm, fall asleep if undisturbed and follow verbal commands. An ideal sedation regime should provide patient comfort, cooperation, amnesia, hemodynamic stability, blunt airway reflexes and maintain a patent airway with spontaneous ventilation. Available conventional sedatives such as benzodiazepines, opioids and propofol cause respiratory depression, especially when used in higher doses. Dexmedetomidine, an $\alpha 2$-adrenoreceptor agonist, is a valuable drug for fiber optic intubation as it induces sedation and analgesia without depressing respiratory function ${ }^{(2)}$.

In addition, xerostomia is commonly reported by patients. These two effects make dexmedetomidine highly desirable for awake fiberoptic nasotracheal intubation ${ }^{(3)}$.

Conscious sedation is achieved by injection of dexmedetomidine at $6 \mathrm{mcg} / \mathrm{kg} / \mathrm{hr}$. Sedation is assessed with the Richmond agitation-sedation scale (RASS) ${ }^{(4)}$. After 10 minutes of dexmedetomidine infusion, the RASS score was 0 to -1 , and the dose of dexmedetomidine was decreased to $0.4 \mathrm{mcg} / \mathrm{kg} / \mathrm{hr}$. The fiberoptic bronchoscope was inserted through the ET tube or before ET tube insertion (the endotracheal tube inserted over the endoscope with the objective of rail roading it). The epiglottis and vocal cords were visualized, and an attempt was made to insert the ET 
tube. When the ET tube reach the vocal cords, the patient starts coughing. After waiting for opening of the vocal cords, $2 \%$ lidocaine was sprayed to the vocal cords through the port of the bronchoscope. When the vocal cords were opened, intubation proceed without difficulty. The position of the ET tube was further confirmed by checking bilateral equal air entry and end-tidal $\mathrm{CO}_{2}$ tracing. The ET tube was then fixed. After tracheal intubation, the nasotracheal tube was secured and general anesthesia was administered. Then dexmedetomidine infusion was stopped and the patient was kept on a mechanical ventilator, allowing the surgeon to start the surgery.

Major challenges during AFOI were providing adequate sedation, maintaining a patent airway and ensuring adequate spontaneous ventilation. Several classes of drugs including benzodiazepines, alpha2 agonists, propofol, ketamine \& ketofol (Ketofol is a combination of ketamine and propofol). To date, there is significant interest in ketofol as an agent for procedural sedation and analgesia. The combination of propofol and ketamine has several benefits in the terms of hemodynamic stability where absence of respiratory depression have been reported for conscious sedation during $\mathrm{AFOI}{ }^{(4)}$.

Among them, dexmedetomidine is a highly selective, potent alpha2 adrenergic receptor agonist. It has the ability to produce profound sedation without causing respiratory depression. In addition, dexmedetomidine decreases salivary secretion through sympatholytic and vagomimetic effects, which is advantageous for fiberoptic intubation ${ }^{(5)}$. Furthermore, dexmedetomidine provided an optimal intubating condition, less hemodynamic instability and better patient tolerance ${ }^{(6)}$. Dexmedetomidine thus has many properties that make it suitable for AFOI, and it has been highly recommended for AFOI.

Also, magnesium $(\mathrm{Mg})$ plays a fundamental role in many physiological process, for example neuronal activity, muscular contraction, and control of vasomotor tone. $\mathrm{Mg}$ is known to possess muscle relaxing effects, mostly by reducing, acetylcholine release, and it seems to have antinociceptive and anesthetic effects which can facilitate fiberoptic nasotracheal intubations ${ }^{(7)}$.

\section{SUBJECTS AND METHODS}

Case control, randomized controlled study in predicted difficult airway patients was carried out at AL-Azhar University Hospitals.
After approval by the local ethical committee, 120 patients of age group 18-60 years old, with American Society of Anesthesiologists I and II criteria were posted for elective surgery under general anesthesia. They were scheduled for awake fiberoptic nasotracheal intubation under conscious sedation after giving their informed written consent to participate in our study. They were randomly divided into four groups, each group included 30 patients: Groups I: received dexmedetomidine bolus $(1 \mathrm{mcg} / \mathrm{kg}$ over10min) followed by a continuous infusion of dexmedetomidine $(0.5 \mathrm{mcg} / \mathrm{kg} / \mathrm{h})$ upon completion of the dexmedetomidine bolus. Group II: received ketofol: (15 $\mathrm{mg}$ ketamine plus propofol) $(100 \mathrm{mic} / \mathrm{kg} / \mathrm{min})$ over $10 \mathrm{~min}$. Group III: received magnesium sulphate $\left(45 \mathrm{mg} / \mathrm{kg} \mathrm{MgSO}_{4}\right.$ in $100 \mathrm{ml}$ of $0.9 \%$ normal saline through $10 \mathrm{~min})$. Group IV (control group): received midazolam $(0.05 \mathrm{mg} / \mathrm{kg} \mathrm{IV})$ in 2-3 minute titrating the dose depending on the patient's response.

Sedation score was assessed following the bolus doses by anesthesiologist unaware of regime used by modified observer assessment of alertness/sedation (OAA/S) scale.

Inclusion criteria: Age 18 to 60 years old of both sexes. ASA physical state I and II.

Exclusion criteria: ASA physical state $\geq$ 3. Dental abscesses. Patient with sever airway trauma, infectious and toxic conditions of the neck and airway. Nasal pathology like nasal polyps. Cardiac patients: A-V block, heart failure, severe bradycardia. Coagulation disorders: liver cirrhosis, thrombocytopenia. Respiratory disorders (COPD, Asthmatic). Uncooperative patients.

Methods: each enrolled patient in this study was subjected to the following: History and physical examination. Airway assessment using El-Ganzouri risk index score.

Investigations: $\mathrm{CBC}$, coagulation profile, ECG and chest X-ray. In addition, other investigations were done as required for each patient.

Patient monitoring: 1- Pulse oximetry (Sp O $\left.\mathrm{O}_{2}\right)$. 2- ECG. 3- NIBP.

The following were assessed: (I) Intubation scores as assessed by: 1-Vocal cord movement (1= open, 2 = moving, $3=$ closing, $4=$ closed). 2 - Coughing $(1=$ none, $2=$ one gag or cough only, $3=>1$ gag or cough, but acceptable conditions, $4=$ unacceptable conditions). 3- Patient tolerance as assessed by facial 
grimace score $(1=$ no grimace, $2=$ minimal grimace, 3 $=$ mild grimace, $4=$ moderate grimace, $5=$ severe grimace, $6=$ very severe grimace). Hemodynamic variables: $\mathrm{HR}, \mathrm{MAP}$ and $\mathrm{Sp} \mathrm{O}_{2}$ were assessed at five different time intervals (baseline, 2 min after sedation, at the beginning of fiberscopy as it passes through the nostril, after or before advancing the ETT through the nasopharynx and 2 min after endotracheal intubation). Intubation time: which is the time from insertion of the fiber optic bronchoscope to completion of intubation. Sedation score: modified observer assessment of alertness/sedation (OAA/S) scale. Procedure adverse events and patient satisfaction.

\section{Statistical analysis:}

Recorded data were analyzed using the statistical package for social sciences, version 20.0 (SPSS Inc., Chicago, Illinois, USA). Quantitative data were expressed as mean \pm standard deviation (SD). Qualitative data were expressed as frequency and percentage. One-way analysis of variance (ANOVA) was used when comparing between more than two means. Chisquare (X2) test of significance was used in order to compare proportions between two qualitative parameters. The confidence interval was set to $95 \%$ and the margin of error accepted was set to $5 \%$. So, the $\mathrm{p}$-value was considered significant as the following: P-value $\leq 0.05$ was considered significant. P-value $\leq 0.001$ was considered as highly significant. P-value $>0.05$ was considered insignificant.

\section{RESULTS}

Table (1): Comparison between groups according to demographic data.

\begin{tabular}{|l|c|c|c|c|c|c|}
\hline \multicolumn{1}{|c|}{ Data } & $\begin{array}{c}\text { DEX } \\
(\mathbf{N = 3 0})\end{array}$ & $\begin{array}{c}\text { Ketofol } \\
(\mathbf{N = 3 0})\end{array}$ & $\begin{array}{c}\text { MgSo4 } \\
(\mathbf{N = 3 0})\end{array}$ & $\begin{array}{c}\text { MID 7BV } \\
(\mathbf{N = 3 0})\end{array}$ & F/x2\# & p-value \\
\hline $\begin{array}{l}\text { Sex: (Female } \backslash \\
\text { Male) }\end{array}$ & $15 \backslash 15$ & $17 \backslash 13$ & $16 \backslash 14$ & $20 \backslash 10$ & $1.900 \#$ & 0.593 \\
\hline $\begin{array}{l}\text { Age (years): Mean } \\
\text { I SD }\end{array}$ & $\begin{array}{c}39.03 \pm \\
10.28\end{array}$ & $\begin{array}{c}34.93 \pm \\
13.90\end{array}$ & $\begin{array}{c}39.27 \pm \\
9.33\end{array}$ & $\begin{array}{c}37.93 \pm \\
9.90\end{array}$ & 0.984 & 0.403 \\
$\begin{array}{l}\text { ASA: (ASA I } \backslash \\
\text { ASA II) }\end{array}$ & $19 \backslash 11$ & $22 \backslash 8$ & $21 \backslash 9$ & $20 \backslash 10$ & $0.770 \#$ & 0.857 \\
\hline
\end{tabular}

DEX= Dexmedetomidine, $\mathrm{MID}=$ Midazolam, \# Fisher exact test

This table showed no statistically significant difference between groups as regards the demographic data ( $\mathrm{p}$ value $>0.05$ ).

Table (2): Elganzori airway score.

\begin{tabular}{|c|c|c|c|c|c|c|}
\hline Airway assessment & $\begin{array}{c}\text { DEX } \\
(\mathbf{N}=30)\end{array}$ & $\begin{array}{c}\text { Ketofol } \\
(\mathbf{N}=30)\end{array}$ & $\begin{array}{c}\text { MgSo4 } \\
(\mathbf{N}=30)\end{array}$ & $\begin{array}{c}\text { MID 7BV } \\
(\mathbf{N}=30)\end{array}$ & F/x2\# & $\begin{array}{c}\mathbf{p}- \\
\text { value }\end{array}$ \\
\hline $\begin{array}{c}\text { Elganzori } \\
\text { airway score: (5 6) })\end{array}$ & $20 \backslash 10$ & $15 \backslash 15$ & $17 \backslash 13$ & $18 \backslash 12$ & 1.783 & 0.619 \\
\hline
\end{tabular}

\# Fisher exact test
Table (2) demonstrated that there was no significant difference regarding Elganzori airway score ( $\mathrm{p}$ value $>0.05$ )

Table (3): Comparison between groups according to tolerance (facial grimace).

\begin{tabular}{|c|c|c|c|c|c|c|}
\hline Facial grimace & $\begin{array}{c}\text { DEX } \\
(\mathrm{N}=30)\end{array}$ & $\begin{array}{l}\text { Ketofol } \\
(\mathrm{N}=30)\end{array}$ & $\begin{array}{l}\text { MgSo4 } \\
(\mathrm{N}=30)\end{array}$ & $\begin{array}{c}\text { MID } \\
(\mathbf{N}=30)\end{array}$ & F/x2\# & p-value \\
\hline No grimace 1 & $7(23.3 \%)$ & $0(0.0 \%)$ & $0(0.0 \%)$ & $0(0.0 \%)$ & \multirow{4}{*}{$32.377 \#$} & \multirow{4}{*}{$<0.001$ ** } \\
\hline Minimal grimace2 & $11(36.7 \%)$ & $8(26.7 \%)$ & $6(20.0 \%)$ & \begin{tabular}{|l|}
$4(13.3 \%)$ \\
\end{tabular} & & \\
\hline Mild grimace 3 & $10(33.3 \%)$ & $10(33.3 \%)$ & $10(33.3 \%)$ & \begin{tabular}{|l|}
$8(26.7 \%)$ \\
\end{tabular} & & \\
\hline Moderate grimace 4 & $2(6.7 \%)$ & $12(40.0 \%)$ & $11(36.7 \%)$ & \begin{tabular}{|l|}
$14(46.7 \%)$ \\
\end{tabular} & & \\
\hline Mean \pm SD & $2.23 \pm 0.60$ & $3.13 \pm 0.85$ & $2.87 \pm 0.77$ & $2.93 \pm 0.79$ & 6.291 & $<0.001^{* *}$ \\
\hline
\end{tabular}

This table showed statistically significant differences between groups in regard to facial grimace (between DEX group and MID group with $\mathrm{p}$ value $<0.001)$.

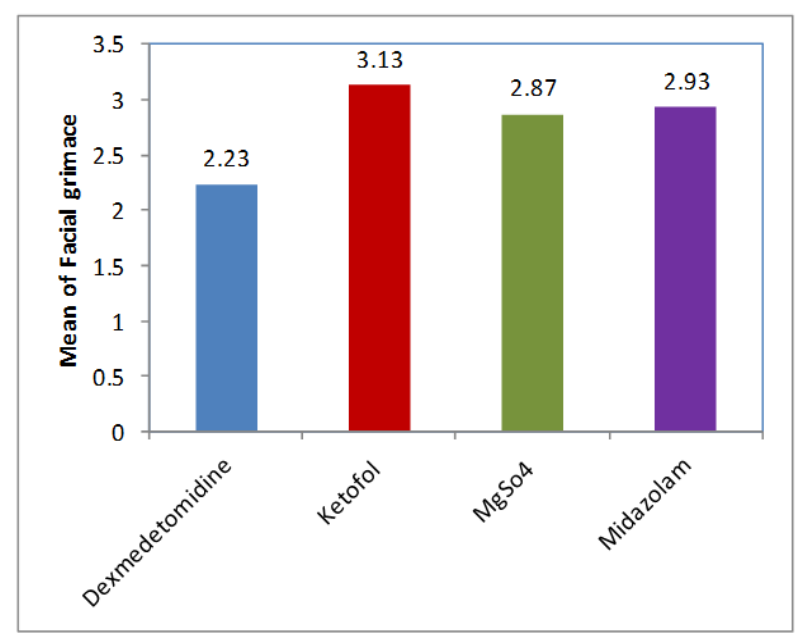

Fig. (1): Bar chart between groups regarding patients' facial grimace.

Table (4): Comparison between groups according to tolerance of (coughing).

\begin{tabular}{|c|c|c|c|c|c|c|}
\hline Coughing & $\begin{array}{c}\text { DEX } \\
(\mathbf{N}=\mathbf{3 0})\end{array}$ & $\begin{array}{l}\begin{array}{l}\text { Ketofol } \\
(\mathrm{N}=30)\end{array} \\
\end{array}$ & $\begin{array}{l}\text { MgSo4 } \\
(\mathrm{N}=30)\end{array}$ & $\begin{array}{c}\text { MID } \\
(\mathrm{N}=\mathbf{3 0})\end{array}$ & $\mathrm{F} / \mathrm{x} 2 \#$ & p-value \\
\hline None1 & $5(16.7 \%)$ & $1(3.3 \%)$ & $1(3.3 \%)$ & $1(3.3 \%)$ & \multirow{4}{*}{ 13.327\# } & \multirow{4}{*}{$0.027 *$} \\
\hline $\begin{array}{l}\text { One gag or cough } \\
\text { only2 }\end{array}$ & $21(70.0 \%)$ & $18(60.0 \%)$ & $16(53.3 \%)$ & $12(40.0 \%)$ & & \\
\hline $\begin{array}{l}>1 \text { gag or cough, } \\
\text { but acceptable } \\
\text { conditions } 3\end{array}$ & $4(13.3 \%)$ & $10(33.3 \%)$ & $12(40.0 \%)$ & $15(50.0 \%)$ & & \\
\hline $\begin{array}{l}\text { Unacceptable } \\
\text { conditions } 4\end{array}$ & $0(0.0 \%)$ & $1(3.3 \%)$ & $1(3.3 \%)$ & $2(6.7 \%)$ & & \\
\hline Mean \pm SD & $1.97 \pm 0.53$ & $2.37 \pm 0.64$ & $2.43 \pm 0.66$ & $2.60 \pm 0.70$ & 4.196 & $0.034 *$ \\
\hline
\end{tabular}

\# Fisher exact test, *: significant

This table (4) showed statistically significant difference between DEX and MID groups regarding coughing ( $\mathrm{p}$ value $<0.05$ ). 


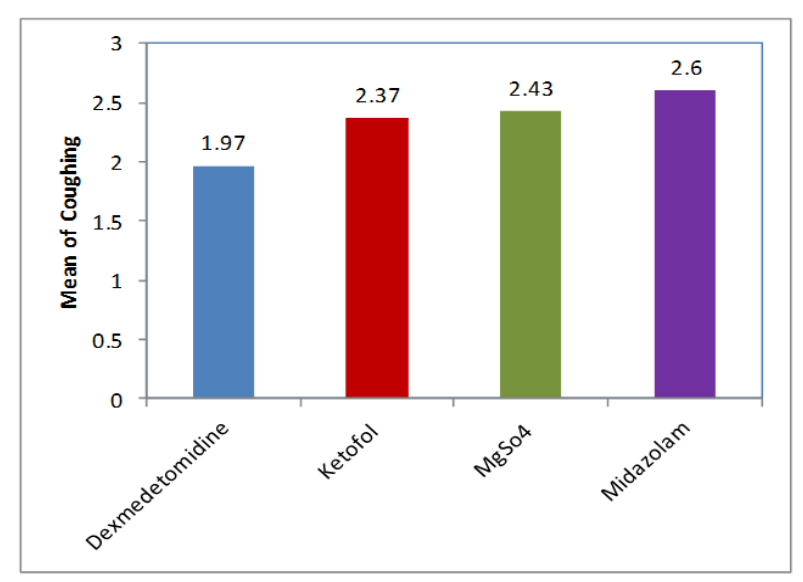

Fig. (2): Bar chart between groups about patients' coughing.

Table (5): Comparison between groups concerning patients' tolerance.

\begin{tabular}{|l|c|c|c|c|c|c|}
\hline $\begin{array}{c}\text { Patients } \\
\text { tolerance }\end{array}$ & $\begin{array}{c}\text { DEX } \\
(\mathbf{N}=\mathbf{3 0})\end{array}$ & $\begin{array}{c}\text { Ketofol } \\
(\mathbf{N}=30)\end{array}$ & $\begin{array}{c}\text { MgSo4 } \\
(\mathbf{N}=30)\end{array}$ & $\begin{array}{c}\text { MID } \\
(\mathbf{N}=30)\end{array}$ & F & $\begin{array}{c}\text { p- } \\
\text { value }\end{array}$ \\
\hline Mean \pm & $2.10 \pm$ & $2.75 \pm$ & $2.65 \pm$ & $2.77 \pm$ & 3.17 & 0.022 \\
SD & $0.57 \mathrm{~b}$ & $0.75 \mathrm{a}$ & $0.72 \mathrm{a}$ & $0.75 \mathrm{~A}$ & 6 & $*$ \\
\hline
\end{tabular}

*: significant, A: control, a: non signfincant with control, b: signfincant with control

This table (5) showed statistically significant difference between groups according to patients' tolerance.

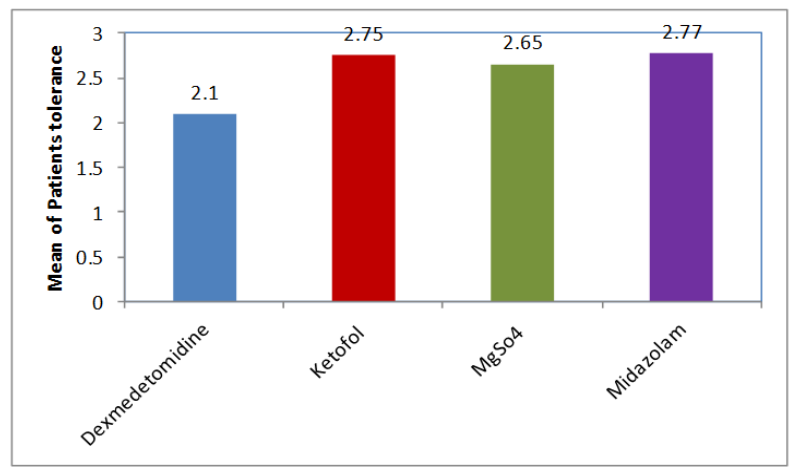

Fig. (3): Bar chart between groups according to patients' tolerance.

Table (6): Comparison between groups about MAP.

\begin{tabular}{|c|c|c|c|c|c|c|}
\hline MAP & $\begin{array}{c}\text { DEX } \\
(\mathrm{N}=30)\end{array}$ & $\begin{array}{l}\text { Ketofol } \\
(\mathrm{N}=30)\end{array}$ & $\begin{array}{l}\text { MgSo4 } \\
(\mathrm{N}=30)\end{array}$ & $\begin{array}{c}\text { MID } \\
(\mathbf{N}=30)\end{array}$ & ANOVA & \begin{tabular}{|c|} 
p- \\
value \\
\end{tabular} \\
\hline $\begin{array}{l}\text { Base line: } \\
\text { Mean } \pm \text { SD } \\
\end{array}$ & $87.63 \pm 5.40$ & $86.10 \pm 6.04$ & $85.40 \pm 8.28$ & $85.73 \pm 6.58$ & 1.177 & 0.177 \\
\hline \begin{tabular}{|l|}
$2-3$ min. after $\mathrm{s}$ \\
edation
\end{tabular} & $.73 \pm 3.74$ & $7 \pm 6.12$ & $83.20 \pm 6.72$ & \pm 4.99 & 1.668 & \\
\hline $\begin{array}{l}\text { During passing } \\
\text { through nostril }\end{array}$ & $\# 81.63 \pm 3.88$ & $82.73 \pm 5.18$ & $83.80 \pm 5.50$ & $83.13 \pm 6.75$ & 1.033 & \\
\hline ced ETT & $\# 80.93 \pm 2.97 \mathrm{~b}$ & $83.13 \pm 6.50 \mathrm{a}$ & $84.67 \pm 11.62 \mathrm{a}$ & $84.27 \pm 6.58 \mathrm{~A}$ & 2.425 & .033 \\
\hline $\begin{array}{l}2-3 \text { min after } \\
\text { intubation }\end{array}$ & $\# 79.13 \pm 4.39 \mathrm{~b}$ & $82.70 \pm 7.41 \mathrm{a}$ & $83.00 \pm 10.14 \mathrm{a}$ & $82.60 \pm 6.54 \mathrm{~A}$ & 3.494 & 0.0 \\
\hline
\end{tabular}

\# Statistically significant compared to baseline, *: significant, A: control, a: non signfincant with control, b: signfincant with control
This table showed statistically significant difference between groups concerning MAP after advanced ETT and 2-3 min after intubation. Statistically significant decrease over the periods through baseline and 2-3 min after sedation, during passing through nostril, after advanced ETT and 2-3 min after intubation (with p-value 0.033, 0.021, 0.015 and 0.010 respectively) using paired sample t-test.

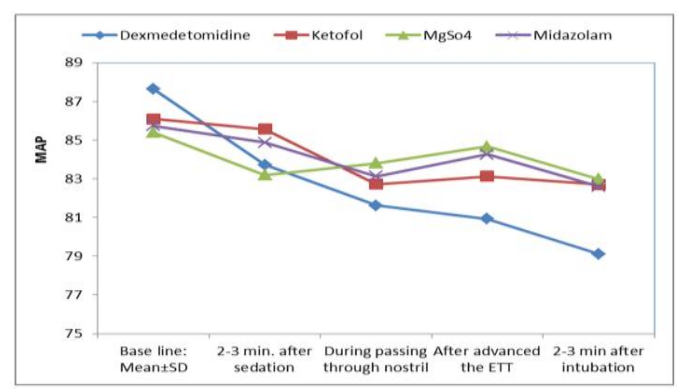

Fig. (4): Line shows the difference between groups regarding MAP.

Table (7): Comparison between groups according to HR (b/min).

\begin{tabular}{|l|c|c|c|c|c|c|}
\hline HR $(\mathbf{b} / \mathbf{m i n})$ & $\begin{array}{c}\text { DEX } \\
(\mathbf{N}=\mathbf{3 0})\end{array}$ & $\begin{array}{c}\text { Ketofol } \\
(\mathbf{N}=\mathbf{3 0})\end{array}$ & $\begin{array}{c}\text { MgSo4 } \\
(\mathbf{N = 3 0})\end{array}$ & $\begin{array}{c}\text { MID } \\
(\mathbf{N = 3 0})\end{array}$ & ANOVA & $\begin{array}{c}\text { p- } \\
\text { value }\end{array}$ \\
\hline Base line & $75.80 \pm 5.14$ & $75.77 \pm 3.74$ & $75.67 \pm 5.23$ & $74.67 \pm 6.01$ & 1.073 & 0.087 \\
\hline $\begin{array}{l}\text { 2-3 min. } \\
\text { after sedation }\end{array}$ & $72.90 \pm 5.01$ & $74.13 \pm 3.67$ & $73.47 \pm 1.81$ & $73.13 \pm 6.39$ & 1.116 & 0.220 \\
\hline $\begin{array}{l}\text { During passing } \\
\text { through nostril }\end{array}$ & $70.63 \pm 4.94$ & $73.43 \pm 3.69$ & $72.87 \pm 5.96$ & $72.40 \pm 6.31$ & 2.073 & 0.384 \\
\hline $\begin{array}{l}\text { After advanced } \\
\text { the ETT }\end{array}$ & $69.03 \pm 5.16 \mathrm{~b}$ & $76.17 \pm 3.82 \mathrm{a}$ & $74.13 \pm 10.23 \mathrm{a}$ & $76.87 \pm 8.38 \mathrm{a}$ & 4.236 & $0.020^{*}$ \\
\hline $\begin{array}{l}\text { 2-3 min after } \\
\text { intubation\# }\end{array}$ & $68.13 \pm 6.47 \mathrm{~b}$ & $72.20 \pm 3.86 \mathrm{a}$ & $71.67 \pm 7.47 \mathrm{a}$ & $71.33 \pm 6.19 \mathrm{~A}$ & 6.106 & $0.027^{*}$ \\
\hline
\end{tabular}

\#Statistically significant compared to baseline, *: significant, A: control, a: non signfincant with control, b: signfincant with control

This table showed statistically significant difference between groups in regard to HR after advanced ETT and 2-3 min after intubation. Statistically significant decrease over the periods through baseline and during passing through nostril, after advanced ETT and 2-3 min after intubation (with p-value $0.029,0.023$ and 0.015 respectively) using paired sample t-test.

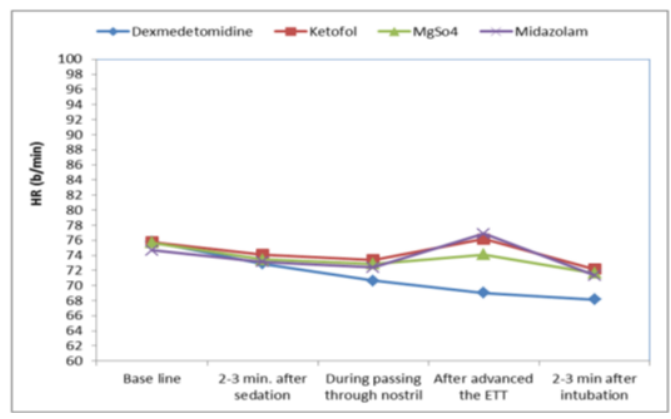

Fig. (5): Line shows the difference between groups concerning $\mathrm{HR}$. 
Table (8): Comparison between groups about $\mathrm{SPO}_{2} \%$.

\begin{tabular}{|l|c|c|c|c|c|c|}
\hline & $\begin{array}{c}\text { DEX } \\
(\mathbf{N}=\mathbf{3 0})\end{array}$ & $\begin{array}{c}\text { Ketofol } \\
(\mathbf{N = 3 0})\end{array}$ & $\begin{array}{c}\mathbf{M g S O}_{4} \\
(\mathbf{N = 3 0})\end{array}$ & $\begin{array}{c}\text { MID } \\
(\mathbf{N}=\mathbf{3 0})\end{array}$ & ANOVA & $\begin{array}{c}\text { p- } \\
\text { value }\end{array}$ \\
\hline Base line & $98.07 \pm 1.01$ & $98.50 \pm 0.68$ & $98.13 \pm 0.63$ & $97.40 \pm 0.81$ & 1.867 & 0.163 \\
\hline $\begin{array}{l}\text { 2-3 min. } \\
\text { after sedation }\end{array}$ & $95.33 \pm 5.66$ & $96.03 \pm 4.69$ & $97.67 \pm 0.71$ & $94.87 \pm 0.63$ & 1.811 & 0.158 \\
\hline $\begin{array}{l}\text { During passing } \\
\text { through nostril }\end{array}$ & $95.10 \pm 5.49$ & $95.83 \pm 4.62$ & $98.07 \pm 0.87$ & $94.18 \pm 0.90$ & 1.539 & 0.134 \\
\hline $\begin{array}{l}\text { After advanced } \\
\text { the ETT }\end{array}$ & $97.77 \pm 0.97$ & $96.90 \pm 2.73$ & $99.00 \pm 0.64$ & $95.00 \pm 0.74$ & 1.308 & 0.114 \\
\hline $\begin{array}{l}\text { 2-3 min after } \\
\text { intubation }\end{array}$ & $98.73 \pm 0.45$ & $98.67 \pm 0.55$ & $99.20 \pm 0.55$ & $96.60 \pm 0.50$ & 1.693 & 0.148 \\
\hline
\end{tabular}

*: significant, A: control, a: non signfincant with control, b: signfincant with control

This table showed no statistically significant difference between groups concerning $\mathrm{SPO}_{2} \%$.

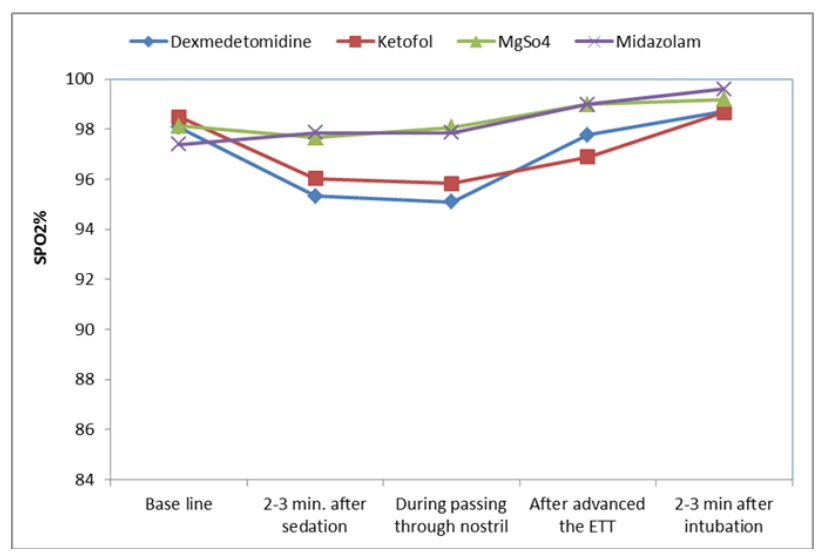

Fig. (6): Line shows the difference between groups concerning $\mathrm{SPO}_{2} \%$.

Table (9): Comparison between groups according to fiber optic view (vocal cords movement).

\begin{tabular}{|l|c|c|c|c|c|c|}
\hline $\begin{array}{l}\text { Fiber optic } \\
\text { view (vocal } \\
\text { cord } \\
\text { movement) }\end{array}$ & $\begin{array}{c}\text { DEX } \\
\text { (N=30) }\end{array}$ & $\begin{array}{c}\text { Ketofol } \\
(\mathbf{N = 3 0 )}\end{array}$ & $\begin{array}{c}\text { MgSo4 } \\
(\mathbf{N = 3 0})\end{array}$ & $\begin{array}{c}\text { MID } \\
(\mathbf{N}=\mathbf{3 0})\end{array}$ & x2 & $\begin{array}{c}\text { p- } \\
\text { value }\end{array}$ \\
\cline { 1 - 5 } Open1 & $13(43.3 \%)$ & $5(16.7 \%)$ & $6(20 \%)$ & $5(16.7 \%)$ & & \\
\hline Moving2 & $15(50 \%)$ & $18(60 \%)$ & $18(60 \%)$ & $19(63.3 \%)$ & 9.496 & 0.148 \\
\hline Closing3 & $2(6.7 \%)$ & $7(23.3 \%)$ & $6(20 \%)$ & $6(20 \%)$ & & \\
\hline Mean \pm SD & $1.83 \pm 0.44$ & $2.07 \pm 0.56$ & $2.00 \pm 0.54$ & $2.03 \pm 0.55$ & 1.692 & 0.254 \\
\hline
\end{tabular}

This table showed no statistically significant difference between groups according to fiber optic view (vocal cord movement).

Table (10): Comparison between groups about intubation time.

\begin{tabular}{|c|c|c|c|c|c|c|}
\hline $\begin{array}{c}\text { Intubation } \\
\text { time/min }\end{array}$ & $\begin{array}{c}\text { Dexmedetomidine } \\
(\mathbf{N}=30)\end{array}$ & $\begin{array}{c}\text { Ketofol } \\
(\mathbf{N}=30)\end{array}$ & $\begin{array}{c}\mathbf{M g S O}_{4} \\
(\mathbf{N}=\mathbf{3 0})\end{array}$ & $\begin{array}{c}\text { Midazolam } \\
(\mathbf{N}=30)\end{array}$ & ANOVA & $\begin{array}{c}\mathbf{p}- \\
\text { value }\end{array}$ \\
\hline Mean \pm SD & $6.94 \pm 0.45$ & $7.11 \pm 0.35$ & $7.25 \pm 0.19$ & $7.16 \pm 0.41$ & 1.922 & 0.086 \\
\hline
\end{tabular}

This table showed no statistically significant difference between groups about intubation time.
Table (11): Comparison between groups regarding no. of attempts.

\begin{tabular}{|c|c|c|c|c|c|c|}
\hline $\begin{array}{c}\text { No. of } \\
\text { attempts }\end{array}$ & \begin{tabular}{|c} 
Dexmedetomidine \\
$(\mathrm{N}=30)$
\end{tabular} & Ketofol $(\mathrm{N}=30)$ & $\begin{array}{l}\mathrm{MgSO}_{4} \\
(\mathrm{~N}=30)\end{array}$ & $\begin{array}{c}\text { Midazolam } \\
(\mathbf{N}=\mathbf{3 0})\end{array}$ & x2 & $\begin{array}{c}\text { p- } \\
\text { value }\end{array}$ \\
\hline One attempts & $20(66.7 \%)$ & $19(63.3 \%)$ & $18(60.0 \%)$ & $16(53.3 \%)$ & \multirow{3}{*}{1.224} & \multirow{3}{*}{0.747} \\
\hline Two attempts & $10(33.3 \%)$ & $11(36.7 \%)$ & $12(40.0 \%)$ & $14(46.7 \%)$ & & \\
\hline Total & $30(100.0 \%)$ & $30(100.0 \%)$ & $30(100.0 \%)$ & $30(100.0 \%)$ & & \\
\hline
\end{tabular}

This table showed no statistically significant difference between groups regarding No. of attempts.

Table (12): Comparison between groups according to sedation score.

\begin{tabular}{|c|c|c|c|c|c|c|}
\hline $\begin{array}{c}\text { Sedation } \\
\text { score: } \\
(\mathrm{OAA} / \mathrm{S})\end{array}$ & $\begin{array}{c}\text { DEX } \\
(\mathbf{N}=\mathbf{3 0})\end{array}$ & $\begin{array}{c}\text { Ketofol } \\
(\mathbf{N}=\mathbf{3 0})\end{array}$ & $\begin{array}{c}\mathbf{M g S O}_{4} \\
(\mathbf{N}=\mathbf{3 0})\end{array}$ & MID (N=30) & $\begin{array}{c}\text { Kruskal } \\
\text { Wallis }\end{array}$ & p-value \\
\hline Mean $\pm \mathrm{SD}$ & $3.91 \pm 0.71^{\mathrm{a}}$ & $3.72 \pm 0.73^{\mathrm{a}}$ & $4.90 \pm 0.37^{\mathrm{b}}$ & $3.60 \pm 0.50^{\mathrm{A}}$ & 19.484 & $<0.001^{* *}$ \\
\hline
\end{tabular}

*: significant, A: control, a: non signfincant with control, b: signfincant with control

The current table (12) showed that there was no significant changes between DEX and Ketofol with MID group. However, there was a highly significant difference between $\mathrm{MgSo} 4$ and MID ( $p$ value $<0.01$ ). In addition, this table showed highly statistically significant difference between groups according to sedation score.

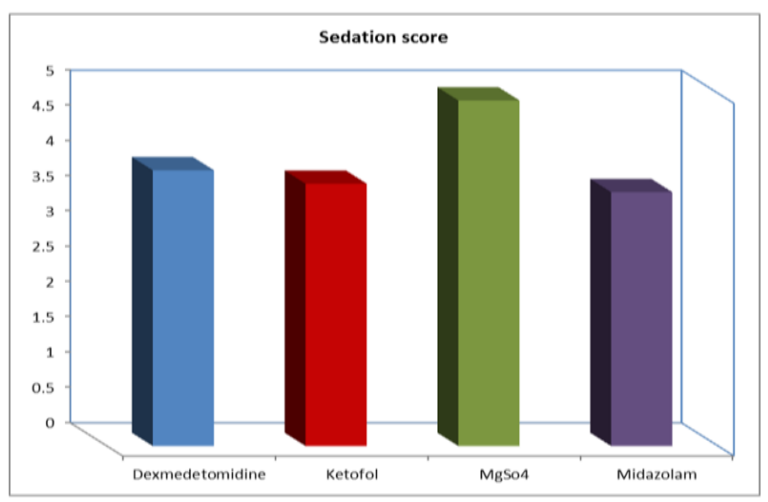

Fig. (7): Bar chart between groups according to sedation score.

Table (13): Comparison between groups concerning adverse events (sore throat/ dysphagia).

\begin{tabular}{|l|c|c|c|c|c|c|}
\hline $\begin{array}{c}\text { Adverse events: } \\
\text { (sore throat/ } \\
\text { dysphagia) }\end{array}$ & $\begin{array}{c}\text { Dexmedetomidine } \\
(\mathbf{N}=\mathbf{3 0})\end{array}$ & $\begin{array}{c}\text { Ketofol } \\
(\mathbf{N}=30)\end{array}$ & $\begin{array}{c}\mathbf{M g S O}_{4} \\
(\mathbf{N}=30)\end{array}$ & $\begin{array}{c}\text { Midazolam } \\
(\mathbf{N}=30)\end{array}$ & $\mathbf{x 2}$ & $\begin{array}{c}\text { p- } \\
\text { value }\end{array}$ \\
\hline Sore throat & $2(6.7 \%)$ & $2(6.7 \%)$ & $4(13.3 \%)$ & $3(10 \%)$ & 1.101 & 0.777 \\
\hline Hypoxic episodes & $0(0 \%)$ & $1(3.3 \%)$ & $0(0 \%)$ & $2(6.7 \%)$ & 3.761 & 0.288 \\
\hline Hoarseness & $2(6.7 \%)$ & $3(10 \%)$ & $5(16.7 \%)$ & $4(13.3 \%)$ & 1.617 & 0.656 \\
\hline
\end{tabular}

This table showed no statistically significant difference between groups concerning adverse events score throat/ dysphagia \& hypoxic episodes. 
Table (14): Comparison between groups according to patients' satisfaction.

\begin{tabular}{|c|c|c|c|c|c|c|}
\hline $\begin{array}{c}\text { Patients } \\
\text { satisfaction }\end{array}$ & $\begin{array}{c}\text { Dexmedetomidine } \\
(\mathbf{N}=\mathbf{3 0})\end{array}$ & $\begin{array}{l}\text { Ketofol } \\
(\mathrm{N}=30)\end{array}$ & $\begin{array}{l}\mathrm{MgSO}_{4} \\
(\mathrm{~N}=30)\end{array}$ & $\begin{array}{c}\text { Midazolam } \\
(\mathrm{N}=\mathbf{3 0})\end{array}$ & x2 & \begin{tabular}{|c|} 
p- \\
value
\end{tabular} \\
\hline Excellent 1 & $4(13.3 \%)$ & $1(3.3 \%)$ & $1(3.3 \%)$ & $1(3.3 \%)$ & \multirow{4}{*}{14.024} & \multirow{4}{*}{0.042} \\
\hline Good 2 & $16(53.3 \%)$ & \begin{tabular}{|l|l}
$10(33.3 \%)$ \\
\end{tabular} & $13(43.3 \%)$ & $8(26.7 \%)$ & & \\
\hline Fair 3 & $9(30 \%)$ & \begin{tabular}{|l|}
$14(46.7 \%)$ \\
\end{tabular} & $17(56.7 \%)$ & \begin{tabular}{|l|}
$17(56.7 \%)$ \\
\end{tabular} & & \\
\hline Poor 4 & $1(3.3 \%)$ & $5(16.7 \%)$ & $2(6.7 \%)$ & $4(13.3 \%)$ & & \\
\hline Mean \pm SD & $2.23 \pm 0.60 \mathrm{~b}$ & $2.77 \pm 0.75 a$ & $2.87 \pm 0.77 \mathrm{a}$ & $2.80 \pm 0.76 \mathrm{a}$ & 3.692 & $0.039^{*}$ \\
\hline
\end{tabular}

This table showed statistically significant difference between groups according to patients' satisfaction (group I vs IV, $\mathrm{p}=0.012 \mathrm{~S}$ ).

\section{DISCUSSION}

Difficult intubation is life threatening condition and was defined as an inability to place an endotracheal tube under direct vision by reasonable experienced anesthetist despite optimal head and neck positioning, very forceful elevation of the laryngoscopic blade, use of multiple attempts with external posterior and cephalad displacement of the larynx, and full paralysis ${ }^{(8)}$.

Difficult intubation usually corresponds to poor glottic visualization during direct laryngoscopy, or a high-grade laryngeal view with inability to see the vocal cords or even part of the glottic aperture.

Airway score 5 or more of El-Ganzouri score indicate a wake intubation and is strongly recommended by any preferred method according to personnal experience, however, fiber optic is preferred ${ }^{(9)}$.

Awake fiber optic intubation (AFOI) is the preferred method for securing a difficult airway. Many agents like midazolam, ketamine, propofol have been used to facilitate FOI, but dexmedetomidine has many properties to make it suitable for use during FOI.

The present study was designed in patients undergoing elective surgery with predicted difficult intubation to compare the effects of dexmedetomedine infusion and magnesium sulphate infusion, ketofol (ketamine propofol combination) in incremental doses compared to midazolam as regard to sedative effects, hemodynamic stability, patient tolerance, intubation time, intubation attempts, patient satisfaction, during AFOI.

The primary outcomes of the study show that dexmedetomidine and ketofol provide satisfactory intubating conditions for AFOI with minimal adverse effects and better patient satisfaction.

In our study satisfactory intubating conditions (facial grimace coughing and patient tolerance) were found in Group DEX. with better tolerance and less facial grimace and less coughing in Group DEX. than other ketofol, magnesium and midazolam groups.

The results of our study showed that dexmedetomidine provides optimum sedation without compromising airway or hemodynamic instability with favorable intubation time and less intubation attempts during $\mathrm{AFOI}$ in comparison other groups.

Dexmedetomidine is a highly selective, centrally acting $\alpha-2$ agonist. It acts on presynaptic $\alpha-2$ receptors to provide negative feedback causing less neurotransmitter (norepinephrine, epinephrine) available at post-synaptic $\alpha-1$ receptors. It produces hypnosis, amnesia, analgesia, anxiolysis, sympatholysis and antisialogogue effects all of which are desirable during AFOI ${ }^{(2)}$.

Dexmedetomidine induces sedation involving activation of endogenous sleep promoting pathway through the post-synaptic $\alpha-2$ receptors in the locus ceruleus, which modulates wakefulness. The primary site of action of alpha2 agonists is the locus ceruleous and not the cerebral cortex, unlike gamma-amino butyric acid- mimetic drugs. Locus ceruleous (nucleus in the pons) that is involved in physiological response to stress and anxiety is the principal site in the brain for norepinephrine synthesis. The major advantages of dexmedetomidine infusion during AFOI are a unique form of sedation where patients remain sleepy, but are easily aroused, cooperative with minimum respiratory impairment. The feasibility of dexmedetomidine has been recently studied either as a sole sedative agent or as an adjuvant during $\mathrm{AFOI}^{(10)}$.

As regard to haemodynamics their was minimal decrease in HR and MAP in all four groups after running of infusion drugs from the base line without haemodynamics instability.

There was deacrease HR in dexmedetomidine group compared to ketofol, magnesium sulphate, and midazolam group at time of intubation and also there was decrease MAP readings in Dexmedetomidine group compared to ketofol, midazolam group and magnesium sulphate group at 
time of intubation because dexmedetomidine infusion may cause bradycardia, hypotension. So in our study the hemodynamic response with dexmedetomidine to midazolam was stable with mild decrease in heart rate response with dexmedetomidine also the blood pressure response was significantly different with a mild depression of blood pressure with dexmedetomidine.

Several authors have also shown a lower heart rate with dexmedetomidine when used as a bolus or infusion due to the vagomimetic and sympatholytic effect of the drug. so this response was observed in our study. It has been also recommended that anticholinergics be used to obviate this effect.

Dexmedetomidine also lowers sympathetic tone. It's mechanism of action lowers fear and anxiety, whereas midazolam inhibits a reaction of the patient to uninhibited stimuli. This may explain why sedation with dexmedetomidine is preferred by many patients over midazolam, which is in line with the crossover study of Ustun et al. ${ }^{(11)}$.

However, some authers observed biphasic state of dexmedetomidine in their study with early hypertension.

Bloor et al. (12) had shown that the hypertension is due to the vasoconstrictive effect of the drug on the blood vessels. This biphasic response has not been uniformly observed in our study and by other authors.

There was mild decrease in MAP after running of the infusion when compared with the baseline MAP in all groups. Also MAP decreased two minutes after intubation in all four Groups that can be attributed to the use of induction agents.

None of the patients in either groups had a fall in the mean HR and MAP more than 20\% from the baseline value.

\section{As regarded to sedation level:}

Dexmedetomidine has been shown to offer adequate conscious sedation for the AFOI. Dexmedetomidine is an alpha-2 adrenoreceptor agonist which acts at the locus coeruleus and produces a conscious sedation due to activation if the endogenous sleep promoting pathway. It has been demonstrated to have anesthetic and analgesic properties in addition to amnesic effects.

However level of sedation in Group dexmedetomedine was slightly lighter than ketofol and midazolam with no satistically significant so dexmedetomidine is a recently introduced for conscious sedation that has been shown to be safe and effective for this purpose.

Midazolam is a sedative and as such depresses the central nervous system; this has the potential to cause cardiac/respiratory depression. The most common undesirable effects are loss of respiratory volume and or fall in respiratory rate or apnoea.

Dexmedetomidine provides appropriate sedation in which the patient is calm and easily arousable from sleep to wakefulness to allow cooperation, excellent communication and task performance while being ventilated and intubated and then quickly back to sleep when not stimulated.

None of the patients were sedated to score less than two (modified AOOIS) score in either the four groups.

Abdelmalak et al. ${ }^{(13)}$ reported aseries of successful awake fibreoptic intubations using dexmedetomidine for sedation in patients with difficult airways caused by a subglottic mass, a thyroid tumour causing tracheal compression, and morbid obesity with sleep apnoea which is correlated with results of our study.

Ketofol is a mixture of ketamine and propofol which provides sedation, analgesia along with hemodynamic stability, which are beneficial for AFOI but there is a risk of hypoxic episodes and respiratory compromise.

Ketofol group had better hemodynamic stability with minimal change in MAP and HR in all time interval (after sedation and during intubation) because of the attenuation of bradycardia and hypotension of propofol by ketamine. The opposing action of ketamine and propofol on cardiac and sympathetic system probably resulted in a more stable hemodynamic response.

Scheinin et al. ${ }^{(14)}$ noted an increase in mean HR during laryngoscopy and intubation; however, we never encountered any increase in the HR, which could probably be related to the use of lidoocaine through "spray as you go" technique in anesthetizing the upper airway.

Also using this drug combination is theoretically compelling, as the sedative effects of 
propofol are thought to counter balance the nauseant and psychomimetic effects of ketamine. The ability to provide sedation with lower doses of ketamine may achieve shorter recovery times compared to use of ketamine alone with larger doses. Moreover, ketamine provides an analgesic effect during propofol infusion that may result in fewer adverse airway events, reduces respiratory depression, and provides more hemodynamic stability ${ }^{(15)}$.

With Ketofol as an induction agent, MAP was maintained while decreased with propofol before intubation. However MAP increased after intubation and before induction of general anesthesia with both drugs due to stress of intubation ${ }^{(16)}$.

As regard $\mathrm{MgSO} 4$ Group: This study showed that IV MgSO4 infusion improved AFOI intubation without marked adverse hemodynamic or respiratory effects.

Dubé et al. ${ }^{(17)}$ showed the mechanism of action of $\mathrm{Mg}$ appears to be multifactorial. It has analgesic, anesthetic and muscle relaxant effects. Also magnesium sulfate being an N-methyl-Daspartate receptor antagonist has both analgesic and sedative properties and has been extensively used in anesthesia.

There was limitation in our study as regards usage of magnesium sulphate as sole agent for sedation, larger doses may be needed to induce sedation, future studies may focus on its use as an adjuvant, or in different doses. furthermore, the effect of those drugs on air way reflexes wasn't studied as we used local anesthetics to blunt them.

However Choi et al. ${ }^{(18)}$ concluded that IV $\mathrm{Mg} \mathrm{SO} 4$ reduced propofol infusion requirements. It could be related to the sedative effect of $\mathrm{Mg}$. $\mathrm{Mg}$ has been reported to produce general anesthesia and to enhance the activity of local anesthetic agents.

Freiberger et al. ${ }^{(19)}$ also showed that Magnesium decreases catecholamine release from the adrenal medulla and adrenergic nerve endings it obtunds the pressor response to laryngoscopy and intubation.

It has been also shown to have antisialogogue effects due to sympatholytic and vagomimetic effects by Kamibayashi et al. ${ }^{(20)}$.
So magnesium sulfate considered an important adjunct to AFOI in patients with suspension of difficult intubation, its role in enhancing patient comfort and cooperation is particularly crucial for procedures performed under local or regional anesthesia.

Moreover, such affective components can complicate the procedure on two levels. The cooperativeness of the patients may be reduced, thereby increasing the risk of complications and making the continuation of the procedure technically difficult. More importantly, they may cause an exaggerated neuroendocrine stress response. It is well established that sympathetic surges can lead to hypertension, tachycardia, ischemic strain on the heart, hyperventilation. These effects are particularly detrimental in elderly patients with multiple comorbidities and lower physiological reserves ${ }^{(21)}$.

As regard to Fiber optic view (vocal cord movement), Intubation attempts and Intubation time showed no statistically significant difference between the four groups which may be related to efficacy of local anaethetics.

As regard to adverse effects like hoarseness of voice and dysphagia post operatively there was no statistically difference between the four groups.

As regard patient Satisfaction there was statistically significant difference between groups according to patients satisfaction. $\mathrm{p}$ value between group DEX. to group midazolam $(p=0.012 \mathrm{~s})$ and there was no statistically significant difference between ketofol, magnesium sulphate, and midazolam. means that dexmedetomedine better satisfaction for patients than other organs.

Bergese et al. ${ }^{(22)}$ in their study of 55 patients undergoing AFOI have shown that Dexmedetomidine treated patients were more satisfied and were calmer and more cooperative during the procedure.

Chu et al. (6) have reported better intubating conditions and patient comfort in patients who received dexmedetomidine. Dexmedetomidine combined with topical anesthesia provided better patient tolerance and amnesia and satisfaction. 
In our study, there was only one hypoxic episodes $(\mathrm{SpO} 2 \leq 90 \%)$ in ketofol group and two episodes in midazolam group.

Kumar ${ }^{(23)}$, found that dexmedetomidine caused no respiratory depression, and this is consistent with our study.

Hense the results of our study showed that dexmedetomidine provides optimum sedation without compromising airway or hemodynamic stability with favorable intubation time and less intubation attempts during AFOI in comparison to magnesium sulphate, ketofol and midazolam patients and also better patient tolerance and satisfaction.

The most important advantage of dexmedetomidine seems to be its ability to exert analgesic and anxiolytic effects without causing respiratory depression or hypoxic episodes.

\section{LIMITATION OF OUR STUDY}

One of the limitations of the study was small sample size. The main limitation of our study is that we did not perform bispectral index (BIS) measurement which an objective technique used for evaluating depth of sedation. We suggest that the incidence of coughing during AFOI should be attributable to inadequate airway topical anaesthesia by short interval of lidocaine spray lidocaine $2 \%$ start to produce topical anesthesia after one minute but need 3-5 minute of contact time to produce maximum penetration of the air way mucosa to produce maximal effect.

\section{CONCLUSION}

From our study, we conclude that all of dexmedetomidine, midazolam, ketofol and magnesium sulphate is effective sedative agent for AFOI when used with "spray as you go technique" for anesthetizing the upper airway with superiorty of Dexmedetomidine as it allows better patient tolerance, better patient satisfaction and acceptable sedative level without any respiratory depression or clinically significant hemodynamic compromise. On the other hand ketofol was good competitive as safe sedative to dexmedetomidine with more hemodynamic stability with accepted fewer side effects. while magnesium sulphate appears not sufficient as solo sedation agent and we recommended to use it as adjuvant to other sedatives.

\section{REFERENCES}

1. Johnston KD, Rai MR (2013): Conscious sedation for awake fibreoptic Intubation: A review of the literature. Can J Anaesth., 60(6): 584-599.

2. Hall JE, Uhrich TD, Barney JA, Arain SR, Ebert TJ (2000): Sedative, amnestic, and analgesic properties of small-dose dexmedetomidine infusions. Anesth Analg., 90(3): 699-705.

3. Maroof M, Khan RM, Jain D, Ashraf M (2005): Dexmedetomidine is a useful adjunct for awake intubation. Can J Anaesth., 52(7): 776-7.

4. Sessler CN, Gosnell MS, Grap MJ, Brophy GM, O'Neal PV, Keane KA, Tesoro EP, Elswick RK (2002): The Richmond Agitation-Sedation Scale: Validity and Reliability in Adult Intensive Care Unit Patients. American Journal of Respiratory and Critical Care Medicine, 166(10): 1338-1344.

5. Belleville JP, Ward DS, Bloor BC, Maze $M$ (1992): Effects of Intravenous Dexmedetomidine in Humans. I.Sedation, Ventilation, and Metabolic Rate. Anesthesiology, 77(6): 1125-1133

6. Chu KS, Wang FY, Hsu HT, Lu IC, Wang HM, Tsai CJ (2010): The Effectiveness of Dexmedetomidine Infusion for Sedating Oral Cancer Patients Undergoing Awake Fibreoptic Nasal Intubation. European Journal of Anaesthesiology, 27(1): 36-40.

7. Herroeder S, Schönherr ME, De Hert SG, Hollmann MW (2011): MagnesiumEssentials for anesthesiologists. Anesthesiology, 114(4):971 -93.

8. Benumof JL and Cooper SD (1996): Quantitative improvement in laryngoscopic view by optimal external laryngeal manipulation. J Clin Anesth., 8:136-140.

9. El-Ganzouri AR, McCarthy RJ, Tuman KJ, Tanck EN, Ivankovich AD (1996): Preoperative airway assessment: predictive value of a multivariate risk index. Anesthesia \& Analgesia, 82(6):1197-204. 
10. Neumann MM, Davio MB, Macknet MR, Applegate RL (2009): Dexmedetomidine for awake fiberoptic intubation in a parturient with spinal muscular atrophy type III for cesarean delivery. Int J Obstet Anesth., 18:403-407.

11. Ustun Y, Gunduz M, Erdogan O, Benlidayi ME (2006): Dexmedetomidine versus midazolam in outpatient third molar surgery. J Oral Maxillofac Surg., 64(9):1353-1358.

12. Bloor BC, Ward DS, Belleville JP, Maze $M$ (1992): Effects of intravenous dexmedetomidine in humans. II. Hemodynamic changes. Anesthesiology, 77(6):1134-42.

13. Abdelmalak B, Marcanthony N, Abdelmalak J, Machuzak MS, Gildea TR, Doyle DJ (2010): Dexmedetomidine for anesthetic management of anterior mediastinal mass. Journal of anesthesia, 24(4):607-610.

14. Scheinin B, Lindgren L, Randell T, Scheinin H, Scheinin M (1992): Dexmedetomidine attenuates sympathoadrenal responses to tracheal intubation and reduces the need for thiopentone and peroperative fentanyl. $\mathrm{Br} \mathrm{J}$ Anaesth., 68(2):126-131.

15. Andolfatto G, Abu-Laban RB, Zed PJ, Staniforth SM, Stackhouse S, Moadebi S, Willman E (2012): Ketamine-propofol combination (ketofol) versus propofol alone for emergency department procedural sedation and analgesia: a randomized double-blind trial. Annals of emergency medicine, 59(6):504-512.

16. Samir J, Coisel Y, Chanques G, Futier E, Constantin JM, Michelet P, Beaussier M, Lefrant JY, Allaouchiche B, Capdevila X, Marret E (2012): A multicentre observational study of intra - operative ventilatory management during general anaesthesia: tidal volumes and relation to body weight. Anaesthesia, 67(9):999-1008.
17. Dubé L and Granry JC (2003): The therapeutic use of magnesium in anesthesiology, intensive care and emergency medicine: A review. Can J Anaesth., 50(7):732-746.

18. Choi JC, Yoon KB, Um DJ, Kim C, Kim JS, Lee SG (2002): Intravenous magnesium sulfate administration reduces propofol infusion requirements during maintenance of propofol-N2O anesthesia: Part I: Comparing propofol requirements according to hemodynamic responses: Part II: Comparing bispectral index in control and magnesium groups. Anesthesiology, 97(5):1137-1141.

19. Mallampati SR, Gatt SP, Gugino LD, Desai SP, Waraksa B, Freiberger D, et al. (1985): A clinical sign to predict difficult tracheal intubation: A prospective study. Can Anaesth Soc J., 32(4):429-434.

20. Kamibayashi $T$ and Maze $M$ (2000): Clinical uses of alpha -2 adrenergic agonists. Anesthesiology, 93(3):13451349.

21. Kumar A, Kaur H, Devi P, Mohan V (2009): Role of coenzyme Q10 (CoQ10) in cardiac disease, hypertension and Menierelike syndrome. Pharmacology \& therapeutics, 124(3):259-268.

22. Bergese SD, Khabiri B, Roberts WD, Howie MB, McSweeney TD and Gerhardt MA (2007): Dexmedetomidine for Conscious Sedation in Difficult Awake Fiberoptic Intubation Cases. Journal of Clinical Anesthesia, 19: 141- 144.

23. Kumar S (2014): A new analytical modelling for fractional telegraph equation via Laplace transform. Applied Mathematical Modelling, 38(13):3154-3163. 